\title{
L'implication des étudiants dans la formation médicale
}

\section{Student involment in medical education}

\author{
Robbert DuvivieR ${ }^{1}$, Maxime Moulin ${ }^{2}$ et Julien DeNIS ${ }^{3}$ \\ 1 Université de Maastricht, Pays-Bas \\ 2 Faculté de médecine de l'Université Pierre et Marie Curie, Paris, France \\ 3 Faculté de médecine Toulouse Rangueil, Université Paul Sabatier, Toulouse, France
}

Manuscrit reçu le 26 octobre 2009 ; commentaires éditoriaux formulés aux auteurs le 18 août 2010 ; accepté pour publication le 31 août 2010

$\begin{array}{ll}\text { Mots clés : } & \text { Résumé - Objectifs : Présenter un panorama général des diverses modalités selon lesquelles } \\ \text { étudiants ; } & \text { les étudiants s'impliquent dans les réflexions et les décisions académiques relatives à l'or- } \\ \text { associations ; } & \text { ganisation des études médicales et présenter les structures institutionnelles dédiées à cette } \\ \text { études médicales ; } & \text { action. Exégèse : Au niveau international, les étudiants en médecine sont regroupés dans } \\ \text { éducation médicale } & \text { la Fédération internationale des associations d'étudiants en médecine (International Federa- } \\ & \text { tion of Medical Students' Associations - IFMSA). Celle-ci entretient de multiples relations } \\ & \text { avec plusieurs organisations dans le domaine de l'enseignement médical, avec l'intention de } \\ & \text { représenter la voix des étudiants au niveau international (Association pour l'éducation médi- } \\ & \text { cale en Europe (Association for medical education in Europe - AMEE), Fédération mondiale } \\ & \text { pour l'enseignement de la médecine (World Federation of Medical Education - WFME). Au } \\ & \text { niveau national français, les étudiants en médecine sont représentés par l'ANEMF (Associa- } \\ & \text { tion nationale des étudiants en médecine de France), dont les activités sont présentées dans } \\ & \text { cet article. } \\ & \text { Abstract - Objectives: To provide an overall view of the various ways in which students } \\ \text { Keywords: } & \text { participate in debates and academic decisions on the organization of medical studies and to } \\ \text { students; } & \text { present institutional structures focusing on this type of action. Analysis: On the international } \\ \text { associates; } & \text { level, medical students are part of the International Federation of Medical Students' Associ- } \\ \text { medical studies; } & \text { ations (IFMSA), which maintains multiple links with medical education organizations with } \\ \text { medical education } & \text { a view to voice the concerns of international students (Association for Medical Education in } \\ & \text { Europe - AMEE, World Federation for Medical Education - WFME). On the national level } \\ & \text { in France, medical students are represented by ANEMF - Association nationale des étudiants } \\ \text { en médecine de France - whose activities are presented in this article. }\end{array}$

\section{Introduction et objectifs}

Les étudiants en médecine sont fortement impliqués dans leur propre formation. Depuis l'institutionnalisation de la formation médicale, leur engagement s'est toujours révélé extrêmement important, que ce soit en matière de planification des programmes, d'évaluation des enseignements et des programmes ou de participation aux décisions, ceci grâce à leur 
représentation, en tant que membres élus ou désignés au sein des conseils de faculté, des comités de programme ou de divers groupes de travail.

De fait, leur contribution se manifeste à tous les niveaux possibles de prise de décision, depuis leur implication de terrain ou leurs mandats plus académiques dans les conseils de leurs facultés respectives, jusqu'à leur engagement dans les organisations internationales. Plusieurs raisons évidentes expliquent cela. Premièrement, les étudiants constituent la première source d'information concernant les programmes et les informations rétro-actives qu'ils fournissent à cet égard contribuent fortement à les faire évoluer et à les améliorer ${ }^{[1]}$. Deuxièmement, les étudiants sont les bénéficiaires des dispositifs de formation et, à ce titre, leur voix doit être entendue ${ }^{[2]}$. Troisièmement, les étudiants ont une forte légitimité en tant que parties prenantes (stakeholders), qui peut contribuer fortement à soutenir les efforts institutionnels de développement et d'innovation pédagogique ${ }^{[3]}$. Enfin, leur participation active à la gestion des programmes et aux prises de décisions est une responsabilité logique dès lors que les étudiants sont censés être des apprenants actifs. Globalement, il est pleinement reconnu que la participation des étudiants en tant que partenaires compétents, actifs et constructifs est une condition importante qui permet d'améliorer la qualité de l'enseignement médical. Cependant, en dépit de ces arguments généralement reconnus, force est de constater que la participation des étudiants dans les processus de prise de décision n'est pas la même partout. Elle varie considérablement selon les pays, les universités, les facultés et même selon les différents programmes au sein d'une même faculté.

Dans cet article, nous examinons de façon plus particulière l'implication des étudiants en médecine dans les programmes de formation médicale initiale, en fournissant quelques exemples de cette participation. Nous décrivons également comment la participation des étudiants est organisée en France et au niveau international.

\section{Participation des étudiants au niveau international}

$\mathrm{Au}$ niveau international, les étudiants en médecine sont regroupés dans la Fédération internationale des associations d'étudiants en médecine (International Federation of Medical Students' Associations IFMSA). C'est une organisation indépendante, non gouvernementale et apolitique d'étudiants en médecine. Actuellement l'IFMSA comprend 100 organisations nationales membres, issues de 93 pays sur six continents et représente plus d'un million d'étudiants en médecine dans le monde entier. L'IFMSA a été fondée en mai 1951 et est gérée par et pour les étudiants en médecine sur une base non lucrative. Elle est officiellement reconnue comme une organisation non gouvernementale (ONG) au sein de l'Organisation des nations unies et reconnue par l'Organisation mondiale de la santé en tant que Forum international des étudiants en médecine. Sa finalité est d'être au service des étudiants en médecine dans le monde entier.

L'IFMSA a été créé aux Pays-Bas avec le statut d'organisation caritative. Elle développe la majorité de ses travaux sur les thèmes suivants grâce à des comités permanents spécifiquement dédiés :

- Santé publique,

- Santé de la reproduction, incluant les problématiques liées au VIH/SIDA,

- Droits de l'Homme et la Paix,

- Échanges entre les professionnels,

- Échanges liés aux activités de recherche,

- Éducation médicale.

Implication de I'IFMSA en éducation médicale

L'implication dans le champ de l'éducation médicale devrait être une préoccupation de chaque étudiant en médecine dans la mesure où elle influence non seulement la qualité des futurs médecins mais aussi la qualité des soins de santé qui seront délivrés dans le futur. L'IFMSA s'appuie sur un groupe de travail dédié, le Comité permanent sur l'éducation 
Tableau I. Missions du Comité permanent sur l'éducation médicale (Standing Committee on Medical Education - SCOME).

Fournir aux étudiants une formation sur différents sujets liés à la problématique de la formation médicale

Faciliter les discussions entre les étudiants et le corps professoral des facultés

Participer à l'évaluation de la formation médicale

Recueillir localement l'opinion des étudiants et favoriser sa prise en compte opérationnelle

Mettre en œuvre des projets, tels que séminaire, conférences, ateliers, cours

Représenter les étudiants dans les bureaux, conseils et comités des facultés et des universités

L'IFMSA, sur un plan général, et le groupe SCOME, sur un plan plus spécifique, interviennent aux trois niveaux, local, national et international.

médicale (Standing Committee on Medical Education - SCOME), dont le but est de contribuer à créer les conditions d'un environnement d'apprentissage optimal pour tous les étudiants en médecine dans le monde. Ce fut l'un des premiers comités créés, dès la fondation de l'IFMSA en 1951. IFMSA-SCOME fonctionne comme un forum de discussion pour les étudiants intéressés par les différents aspects de l'enseignement médical (tableau I), avec l'idée de favoriser l'atteinte de l'excellence en éducation médicale dans le monde entier.

La stratégie la plus prometteuse pour le changement est une approche locale. Même lorsque les étudiants n'ont pas de représentation officielle au sein des organes institutionnels de prise de décision, ils ont les moyens de convaincre les doyens, les professeurs et les autres parties prenantes d'améliorer les dispositifs de formation. Sans qu'il soit nécessaire de changer l'ensemble du programme, de petites modifications ciblées sur diverses composantes des dispositifs de formation peuvent se révéler efficaces pour améliorer le programme dans son ensemble.

Pour faciliter le travail des représentants locaux de l'IFMSA et leur contribution à des améliorations dans leurs propres institutions ou pays, les principales activités organisées au sein de SCOME sont des sessions de formation. La formation porte sur tous les domaines qui relèvent de l'enseignement médical, comme l'évaluation ou l'apprentissage par problèmes.

Les étudiants partagent leurs expériences et des exemples de meilleures pratiques en ligne, grâce à des listes de diffusion et à une plate-forme spécialement conçue de type Wiki (www.ifmsa. org/scome/wiki). Les étudiants peuvent y télécharger des fichiers et y trouver des présentations concernant le système éducatif des différents pays. Ils ont notamment accès aux archives des ressources développées précédemment.

En outre, deux fois par an, 800 étudiants de près de 100 pays se réunissent pour mutualiser leurs informations lors des assemblées générales de l'IFMSA. Entre temps, des réunions régionales sont organisées pour entretenir la motivation et actualiser les informations. Les résultats des réunions sont partagés entre les membres grâce à la plate-forme Wiki et mises à disposition du grand public. L'objectif est de publier les réflexions de la fédération sur des sujets particuliers, sous la forme de déclarations de principe (policy statements) ou de documents de position (position papers). Au cours des dernières années, l'IFMSA s'est fortement impliquée dans la réflexion concernant l'évolution de l'enseignement supérieur européen et la mise en œuvre du processus de Bologne. Ce travail a fait l'objet d'un certain nombre de publications dans des revues exerçant un contrôle éditorial, telles que Medical Teacher ou Medical Education ${ }^{[4-7]}$ Les résultats de ces travaux ont été également été diffusés lors de conférences où des représentants de l'IMFSA ont été invités en qualité d'orateurs principaux, de membres d'une table ronde ou de participants à un symposium.

Un exemple, qui peut être utile pour les lecteurs francophones européens, est celui du tronc commun européen. Avec le souci d'apporter une réponse aux modifications proposées dans l'enseignement supérieur européen, les étudiants en médecine se 
sont accordés quant aux objectifs d'apprentissage à atteindre par tous les médecins diplômés. Ces objectifs de base communs devraient constituer le tronc commun (core curriculum) européen conforme aux dispositions réglementaires européennes. Ce document ${ }^{[8,9]}$ traduit l'opinion des étudiants en médecine relative aux capacités, connaissances et attitudes que les diplômés des facultés de médecine européennes devraient avoir acquis et au regard desquelles ils devraient donc, en conséquence, être évalués.

Le tronc commun comporte 76 objectifs d'apprentissage, regroupés selon les neuf domaines suivants :

- Compétences cliniques,

- Communication,

- Pensée critique,

- Santé et société,

- Apprentissage tout au long de la vie,

- Professionnalisme, responsabilités, développement personnel,

- Enseignement,

- Travail collaboratif,

- Connaissances théoriques.

Ce tronc commun doit être considéré comme un cadre de référence ayant vocation à être adapté et transposé en tenant compte des besoins locaux. Il constitue une base commune visant à maintenir ou même améliorer la qualité de la formation et des soins de santé et à favoriser la mobilité européenne, qui laisse néanmoins chaque faculté libre et responsable du choix de ses approches pédagogiques ou d'éventuels enseignements complémentaires.

La France envisage fortement la mise en place de la réforme dite LMD (licence, master, doctorat) pour le cursus régulier de formation médicale initiale. À cette occasion, la Commission pédagogique nationale des études médicales (CPNEM) réalise un très important travail de déclinaison du cursus en différentes unités d'enseignement, décrites sous forme d'objectifs d'apprentissage. Le souhait de l'IFMSA est que ce travail respecte la cohérence du dispositif en trois cycles du système LMD, dont le but est de faciliter la visibilité et la comparabilité des différents cursus, en ayant à l'esprit que commencer dès maintenant à implanter un tronc commun européen, c'est poser la première pierre d'un enseignement européen de la médecine.

\section{Les actions de l'IFMSA au niveau international}

L'IFMSA coopère avec des organisations dans le domaine de l'enseignement médical, avec l'intention de représenter la voix des étudiants au niveau international. Ces initiatives ont souvent, en retour, un impact au niveau national et local.

Actuellement, l'IFMSA est représentée au conseil d'administration de l'Association pour l'éducation médicale en Europe (Association for medical education in Europe - AMEE) et au conseil de la Fédération mondiale pour l'enseignement de la médecine (World Federation of Medical Education - WFME). L'AMEE promeut un développement méthodique des différentes activités liées à l'éducation médicale et organise annuellement la plus importante conférence mondiale de recherche en pédagogie des sciences de la santé, à l'occasion de laquelle sont discutés et partagés les travaux et expériences développés dans le champ de la formation des professionnels de santé. La WFME est une organisation concernée par tous les aspects de la formation médicale, concernant à la fois le cursus de base, le cursus de formation spécialisée (internat) ou la formation continue. Elle fédère six organisations «continentales » régionales (Afrique, Amérique, Moyen Orient, Asie du Sud-Est, Pacifique Ouest et Europe).

\section{Les étudiants en médecine en France : I'Association nationale des étudiants en médecine de France (ANEMF)}

Association régie par la loi 1901, l'Association nationale des étudiants en médecine de France (ANEMF) travaille pour l'intérêt commun des étudiants en médecine. Indépendante de tout parti politique ou de toute structure confessionnelle, c'est 
une structure qui réunit les 36 associations des étudiants en médecine de France, soit indirectement 80000 étudiants. L'ANEMF travaille, tout comme l'IFMSA, dans de nombreux champs d'activité : démographie médicale, élus locaux, santé, solidarités, échanges internationaux, représentation internationale, tutorats, formation des associatifs (par de l'éducation non formelle), ... autant de champs d'activités variés qui reflètent l'engagement citoyen des étudiants en médecine de France, que l'ANEMF entend favoriser grâce à des actions de coordination, de diffusion et mutualisation et de formation. Malgré ce large éventail d'activités, l'activité principale de l'ANEMF a toujours été centrée sur les études médicales et notamment leurs réformes successives. En relation avec les ministères de tutelle (Ministère de la santé, Ministère de l'enseignement supérieur et de la recherche), cette mission s'applique à formuler des propositions de nature à apporter des solutions aux évolutions complexes de la formation théorique et pratique, en soutenant le point de vue des étudiants. L'ANEMF tire sa légitimité d'une large représentativité, avec des représentants issus de toutes les facultés françaises, et s'appuie l'expertise des membres de son bureau et sur des groupes de travail, dont les productions sont discutées en assemblée plénière nationale.

Les études de médecine sont particulièrement longues mais pas figées pour autant. Elles font l'objet de réformes diverses, portant sur des thématiques très différentes (structure, contenu pédagogique, évaluation, enseignement, passerelles professionnelles, ...). En France les étudiants ne sont traditionnellement pas impliqués au centre des débats dont ils sont pourtant la cible. Ceci ne favorise pas la qualité des réformes, dès lors que les porteurs de celles-ci se privent de la réflexion, des projets et des visions des professionnels de demain. Sans pour autant être réfractaire à tout changement, l'ANEMF est vigilante par rapport à l'ensemble des projets ministériels qui tendraient à vouloir proposer des réformes radicales, en négligeant les conséquences de telles mesures pour les étudiants. Récemment, le projet de réforme de la première année des études de santé (PAES/L1 santé) a fait l'objet de négociations multiples, en se basant toujours sur les problématiques locales.

Véritable partenaire de plusieurs institutions (Commission pédagogique nationale des études médicales, Conférence des doyens, Agence d'évaluation de la recherche et de l'enseignement eupérieur - AERES -, Groupe d'étude et de recherche en anglais de spécialité - GERAS - et autres commissions diverses), l'ANEMF contribue à chercher des solutions pour améliorer les études de médecine : amélioration des stages cliniques d'externat, modification des programmes pédagogiques, intégration du système LMD, instauration de l'anglais dans les études, ... Même si les réflexions sur ces sujets avancent doucement, l'ANEMF reste motrice dans les débats. Désireux d'être à la pointe des réflexions et des problématiques afin d'assurer une qualité maximale à ses réflexions, le bureau national de l'ANEMF se forme constamment en utilisant plusieurs ressources : suivi des travaux de professionnels (experts de Bologne, groupes de travail thématiques tel Medical Education in Europe - MEDINE -, publications de l'AMEE), participation à des conférences françaises sur l'enseignement supérieur, échanges de connaissances avec les pairs (discussion avec les associations nationales du monde entier via l'IFMSA). Le but est de parvenir à une compréhension optimale de chaque problématique, de manière à être la meilleure force de proposition possible.

Une position de l'ANEMF suit classiquement le schéma suivant :

1. Décision du bureau ou de l'assemblée générale de se saisir d'un dossier (par exemple, réforme des épreuves classantes nationales).

2. Travail documentaire réalisé par l'équipe du bureau de l'ANEMF (évaluation au niveau des facultés par son réseau d'étudiants locaux; documentation théorique sur les examens de fin de cursus, recherche de ce qui ce fait dans d'autres pays via le réseau de l'IFMSA...)

3. Sur la base de ces connaissances, le bureau de l'ANEMF facilite des discussions et organise des 
groupes de travail nationaux pour aboutir à des propositions.

4. Les présidents des associations locales, représentatifs de leurs étudiants, votent en assemblée générale une contribution et entérinent la prise de position sur le dossier, en l'amendant si besoin.

5. Ensuite seulement, le bureau de l'ANEMF défend ces positions auprès de l'ensemble des preneurs de décisions (Ministère, CPNEM,...)

Outre ces activités, l'ANEMF développe aussi des actions directes auprès des étudiants : campagnes d'orientation des lycéens, accès facilités aux ressources pédagogiques de référence via l'Université Médicale Virtuelle Francophone (UMVF), etc. Elle s'efforce surtout de favoriser le développement des tutorats qui ne cessent de prendre de l'ampleur, concernant à présent même les externes.

\section{Références}

1. Visser K,. Prince K, Scherpbier A, Van Der Vleuten C, Verwijnen G. Med Teach 1998;20:451-4.

2. Slater C. Student participation in curriculum planning and evaluation. J Med Educ 1969;44:67-8.
3. Sallis E. Total quality management in education. London: Kegan, 1996.

4. Duvivier RJ, Hilgers J, Davaris N, Rodriguez Muñoz D. Implementation of the Bologna Two-Cycle System in Medical Education - the Students' View. Med Teach 2009;31:376-7.

5. Duvivier RJ, Brouwer EE, Weggemans M. Medical Education in Global Health: Student Initiatives in the Netherlands. Med Educ 2010;44:527-30.

6. Duvivier RJ, Weggemans M. Medical Education in Europe: a Commentary from Students. Tijdschrift voor Medisch Onderwijs 2010;9:220-1.

7. Duvivier RJ, Weggemans M. Joint issue TMO/ZMA: Reply from International Medical Students. GMS Z Med Ausbild 2010;27:Doc40.

8. IFMSA, EMSA, Hilgers J, De Roos P. European core curriculum: the students' perspective, Bristol, UK, 10 July 2006. Med Teach 2007;29:270-75.

9. European Core Curriculum - the Students' Perspective. [On-line] Disponible sur : http://wiki.ifmsa. org/scome/index .php?title=European_Core_ Curriculum_-_the_Students\%27_Perspective

Correspondance et offprints : Robbert Duvivier, Université de Maastricht, Pays-Bas.

Mailto : robbertduvivier@gmail.com 